19 Revue d'histoire du XIXe siècle

Société d'histoire de la révolution de 1848 et des

révolutions du XIXe siècle

29 | 2004

Varia

\title{
Apogée et déclin de la Saint Lundi dans la France du XIX ${ }^{\mathrm{e}}$ siècle
}

Robert Beck

(2) OpenEdition

Journals

Édition électronique

URL : http://journals.openedition.org/rh19/704

DOI : $10.4000 /$ rh19.704

ISSN : $1777-5329$

Éditeur

La Société de 1848

Édition imprimée

Date de publication : 1 décembre 2004

Pagination : 153-171

ISSN : 1265-1354

Référence électronique

Robert Beck, "Apogée et déclin de la Saint Lundi dans la France du XIXe siècle », Revue d'histoire du XIXe siècle [En ligne], 29 | 2004, mis en ligne le 07 avril 2008, consulté le 19 avril 2019. URL : http:// journals.openedition.org/rh19/704 ; DOI : 10.4000/rh19.704

Ce document a été généré automatiquement le 19 avril 2019

Tous droits réservés 


\title{
Apogée et déclin de la Saint Lundi dans la France du XIX ${ }^{e}$ siècle
}

\author{
Robert Beck
}

1 Parlez donc aujourd'hui de la Saint Lundi à une personne peu familiarisée avec l'histoire des classes populaires du XIX ${ }^{e}$ siècle - en général, votre interlocuteur vous avouera toute son ignorance. Ignorance frappante cependant, quand on sait que cette vieille coutume $\mathrm{du}$ monde populaire des villes est considérée au XIX ${ }^{\mathrm{e}}$ siècle comme un des grands fléaux sociaux et moraux du siècle. Coutume que les élites religieuses, industrielles et autres cherchent désespérément à combattre pendant des décennies, avant que les circonstances de la fin de ce siècle ne leur accordent définitivement le triomphe sur un élément traditionnel de la culture populaire, jusqu'au point où la mémoire collective va extirper toute trace de cette culture - ce qui explique l'ignorance de votre interlocuteur.

Il s'agit de la coutume de nombreux ouvriers de chômer volontairement le lundi, parfois même quelques jours de suite. En principe, ces ouvriers se rencontrent à la porte de l'atelier, ou sur le chemin du travail dans un «mastroquet». D'autres commencent le travail pour le quitter ensuite vers le midi et passer le reste de la journée du lundi en dehors de l'atelier. Quelquefois, ils se donnent aussi rendez-vous dans une guinguette sans se rendre sur leur lieu du travail. Cette coutume représente une véritable institution dans le monde du travail qu'on peut rencontrer un peu partout en Europe, dans les pays scandinaves et germaniques, en Catalogne comme en Andalousie, en Belgique comme en Angleterre $^{1}$. Il ne s'agit pas non plus d'une invention du XIXe siècle, malgré les affirmations de certaines sources catholiques, y apercevant une machination de la Révolution pour nuire au dimanche, et ainsi à la religion, mais d'une tradition dont les origines se perdent au Moyen Âge ${ }^{2}$. Dès la fin $\mathrm{du} \mathrm{XVI}^{\mathrm{e}}$ siècle, les premières interdictions frappent la coutume de chômer le lundi, par exemple dans le pays de Montbéliard, ce qui ne l'empêche pas de survivre jusqu'à la Révolution et bien au-delà ${ }^{3}$. À la fin du XVII ${ }^{e}$ siècle, époque de suppression d'une vingtaine de fêtes religieuses, les artisans de Paris ont déjà pris l'habitude de chômer le lundi et un mardi sur deux ${ }^{4}$, alors que les 
compagnons imprimeurs de Lyon, vers 1730, ne commencent leur ouvrage qu'à partir de jeudi ${ }^{5}$.

3 Les témoignages concernant la coutume de la Saint Lundi de la fin de l'Ancien Régime circonscrivent un grand Nord en France où ce chômage ne s'est pas seulement maintenu mais a même pris de l'envergure. Elle n'est pas seulement attestée dans les villes mais aussi dans les artisanats des sociétés rurales, à la fois en Bretagne et en Franche-Comté ${ }^{6}$, et elle concerne surtout les métiers issus des structures de la proto-industrie. Les guinguettes, comme à Paris, semblent jouir de la préférence des artisans et des compagnons pour y passer cette journée ${ }^{7}$, de même, mais dans une moindre mesure, les cabarets intra-muros ${ }^{8}$, où artisans et compagnons prolongent les libations du dimanche.

discours rationnel et productiviste que véhicule le calendrier républicain sous la Révolution, ne réussit pas à mettre un terme à la coutume du lundi. Certains artisans parisiens auraient même profité de l'ambiguïté créée par la coexistence de deux calendriers pour fêter à la fois la Saint Lundi et la "Saint Primidi » ${ }^{9}$. Au début du XIX siècle, à une époque d'industrialisation balbutiante, la Saint Lundi est donc bien vivante et bien ancrée dans le monde du travail. Elle va connaître ensuite une évolution diversifiée, au point que ce siècle constituera le moment à la fois de son apogée et de son déclin définitif. L'objectif de ces lignes est donc de suivre cette évolution, de scruter ses conditions, de montrer la vraie nature de cette fête ainsi que ses modifications, ses protagonistes, ses raisons d'être et ses fonctions dans le monde du travail du XIX ${ }^{\mathrm{e}}$ siècle. Il s'agit de montrer le vrai visage de cette vieille coutume populaire, entièrement défigurée par un discours des classes dominantes.

5 Les sources pour bâtir une telle histoire sont assez éparses. Certes, il existe ce discours assez abondant, des élites religieuses et philanthropes surtout. Celles-ci regardent la Saint Lundi à travers un prisme déformant constitué de normes religieuses et bourgeoises, dictées par des objectifs moraux, sociaux, économiques, voire politiques. Ce discours nécessite un traitement bien délicat pour distinguer le réel du fantasmé. Un autre problème de documentation réside dans la concentration de nos connaissances sur le cas de Paris - cette ville, centre en effet de la géographie de la Saint Lundi, est pourtant loin d'en posséder le monopole. Plus que parsemés sont les renseignements sur la pratique de la Saint Lundi dans les villes de province. À part le discours des élites, nous sommes donc obligé de nous contenter des informations "glanées ", ensuite de quelques enquêtes et finalement de quelques documents judiciaires. Écrire l'histoire de la Saint Lundi signifie donc de la bâtir sur une base documentaire assez rudimentaire, pour procéder ensuite par déductions et par suppositions... Écrire l'histoire de la Saint Lundi représente surtout une certaine gageure.

6 La coutume de la Saint Lundi est donc bien vivante chez les artisans, ouvriers et compagnons français au début du XIXe siècle. Un bulletin de police du 14 novembre 1816 constate que les ouvriers restent fidèles à l'« usage ruineux et immoral» de ne pas se mettre à l'ouvrage ce jour-là ${ }^{10}$. Sous la Restauration, filatures et fabriques sont ainsi considérées comme foyers de "corruption» car nulle part on ne chômerait plus régulièrement le lundi ${ }^{11}$.

7 Sans établir de règle générale, la coutume de la Saint Lundi ne se pratique pas de façon homogène dans toutes les régions avant 1848. Le lundi est d'abord fêté à Paris, ensuite dans les régions industrialisées du Nord et de l'Est, de même que dans les villes industrialisées de l'Ouest et du Centre. Lyon, par exemple, malgré sa richesse industrielle, ne semble pas connaître cette coutume, au moins pas dans l'industrie de la soie. Selon 
Adolphe Blanqui en 1849, le chômage du lundi ne serait pas non plus pratiqué dans le Midi, ni à Bordeaux ni à Marseille ${ }^{12}$. D'autres témoignages montrent cependant une extension géographique de la coutume sous la monarchie censitaire - ainsi les papetiers de Thiers ${ }^{13}$, ou encore les ouvriers de Castres qui auraient adopté l'habitude de chômer le lundi depuis que le vin serait devenu moins cher ${ }^{14}$. De même, les jeunes travailleurs de Marseille, à partir des années 1820, n'hésitent plus à chômer plusieurs jours par semaine 15. Les ouvriers de l'industrie textile de Nîmes considèrent le lundi comme un jour de repos ${ }^{16}$. Louis-René Villermé, quant à lui, constate que les ouvriers de la fabrique de Lodève se reposent au moins le lundi après-midi ${ }^{17}$. Au début des années 1850, Armand Audiganne note le progrès de la Saint Lundi à Lyon ${ }^{18}$, ainsi qu'à Mazamet où les premiers tisseurs des métiers « à la Jacquart » auraient importé la coutume de chômer ce jour-là ${ }^{19}$. Le chômage du lundi prend donc de nouvelles dimensions géographiques avant 1848 , s'étendant, sous l'influence d'ouvriers arrivés du Nord de la France, et des mutations dans les rapports sociaux, vers le Midi de la France, en dépit des déclarations de nos observateurs contemporains ${ }^{20}$.

8 L'enquête menée par la Chambre de commerce de Paris en 1860 ne peut cacher un certain triomphalisme en constatant qu'il existe des «habitudes de moralité chaque jour plus goûtées, plus pratiquées dans le milieu de l'atelier, tendant à faire disparaître les traditions du chômage volontaire du lundi... ${ }^{21}$. D'autres témoignages, comme celui de Pierre Vinçard dès 1851, confirment cette tendance pour Paris ${ }^{22}$. Or l'enquête nationale de 1872 sur la situation de la classe ouvrière montre une Saint Lundi tout à fait vivante dans de nombreuses régions : pour ne citer que celles où cette coutume est quasi absente, il faut évoquer un grand Sud-Est, s'étendant de la Savoie vers les départements du Var et des Alpes-Maritimes, ainsi que quelques départements ruraux de l'Ouest (Morbihan, Côtes-du-Nord, Orne, Mayenne). Certes, la même enquête constate aussi un recul du chômage du lundi dans certaines régions où elle ne s'était jamais vraiment enracinée (Languedoc, Auvergne), mais d'autres régions connaissent un nouvel enracinement de la Saint Lundi (Sarthe, Haute-Vienne...), ou son maintien, voire un renouveau. Cette évolution peut même prendre un caractère contrasté dans certains départements, comme en Indre-et-Loire, où la Chambre de commerce de Tours dépeint un chômage du lundi en voie de disparition dans cette ville, alors que ce même chômage vient de passer dans les mœurs des tanneurs de Château-Renault ${ }^{23}$.

9 Avant 1830, le chômage du lundi est en général étroitement lié à celui du dimanche, formant ainsi une unité temporelle. Or le repos dominical entre, après les Trois Glorieuses, dans une sphère de grandes turbulences. Notamment à Paris, mais aussi dans les centres industriels du Nord et de l'Est de la France, où artisans, compagnons et ouvriers travaillent de plus en plus dans la matinée du dimanche, pour consacrer le reste de la journée à leur famille ${ }^{24}$ et pour fêter, le lendemain, le lundi. Le travail du dimanche se développe donc, et avec lui le chômage du lundi: "La plupart des ouvriers qui travaillent le dimanche, se reposent ensuite le lundi... » écrit Théodore-Henri Barrau en $1850^{25}$. En 1872, 63 cas de chômage du lundi, sur les 98 relevés par l'Enquête sur la situation des classes ouvrières ${ }^{26}$, sont liés au travail du dimanche. La combinaison repos du dimanche et repos du lundi ne se retrouve plus que dans les régions catholiques respectueuses du repos du dimanche.

10 En se décrochant ainsi du temps religieux du dimanche, la Saint Lundi se transforme en alternative au repos dominical à une période où ce dernier perd de son caractère festif pour se transformer en simple jour de repos et de famille selon les exigences et les 
normes de la bourgeoisie. Dorénavant, c'est la Saint Lundi qui possède le monopole de la fête pour une partie de la classe ouvrière. Cette concurrence au dimanche chrétien lui attire par conséquent les foudres des publicistes de l'Église, surtout après la Révolution de 1848.

Ayons recours d'abord à une lithographie, intitulée Litanies de Saint Lundi, datant des premières années de la Monarchie de Juillet ${ }^{27}$. Rassemblés autour d'un personnage en habits déchirés, tenant une cruche et un verre de vin, symbolisant la Saint Lundi, on trouve des représentants des métiers du bâtiment, tailleurs, perruquiers, forgerons, tonneliers et imprimeurs. D'autres témoignages évoquent les chapeliers ${ }^{28}$, les ouvriers tisserands en Normandie ${ }^{29}$, ainsi que les tisserands et fileurs du Nord ${ }^{30}$. La Statistique de l'industrie à Paris fournit des informations plus précises: tout d'abord, l'habitude de chômer le lundi existe dans toutes les industries, à la notable exception de celle de l'alimentation. Vu le nombre de métiers pratiquant cette coutume, cette Statistique ne permet pas de dresser une liste exhaustive, mais de déceler des formes spécifiques chez les adeptes de la Saint Lundi. Ainsi les " pièçards ", ouvriers travaillant à la pièce, comme les tapissiers, sont-ils plus facilement tentés de fêter le lundi ${ }^{31}$, ce qui confirme le constat fait par Louis-René Villermé quelques années avant: "...les ouvriers à la tâche ou aux pièces peuvent ordinairement se reposer quand il leur plait ${ }^{32}$. Les ouvriers disposent donc librement de leur temps de travail, ce que dénoncent à la fois Louis-René Villermé, Louis Reybaud et l'imprimeur Jules Claye comme une des raisons de la Saint Lundi ${ }^{33}$.

12 Le montant du salaire joue également un rôle capital. À Thiers, ce sont les ouvriers gagnant les plus forts salaires qui chôment le lundi ${ }^{34}$. La Statistique de l'industrie à Paris constate ainsi pour les ouvriers parisiens que ceux-ci commencent à chômer le lundi à partir d'un salaire quotidien de trois francs ${ }^{35}$. Les exceptions à cette règle sont en effet rares : citons les cordonniers, qui ne gagnent en moyenne que 2,65 francs par jour, mais chez qui le chômage du lundi s'appuie sur une vieille tradition. Est-ce aussi la tradition qui explique la coutume de la Saint Lundi chez les tisserands normands qui, malgré un salaire plus que modeste, ne "peuvent se défendre de chômer le lundi » ${ }^{36}$ ? Mais dans l'ensemble, il s'agit donc d'ouvriers qualifiés et disposant d'un salaire assez élevé qui fêtent la Saint Lundi.

13 Un autre trait commun à de nombreux adeptes du lundi réside dans leur situation de logement - ils logent souvent en garni, à l'exemple des menuisiers et peintres, tailleurs et graveurs de verres, fondeurs de métaux.... Cette circonstance concerne aussi les femmes (comme celles qui travaillent dans l'horlogerie ${ }^{37}$ ). Si la Saint Lundi peut de nouveau s'installer à Lyon, c'est justement la conséquence de la disparition de la solidarité de l'ancien atelier et des liens de dépendance créés par le fait que l'ouvrier est logé et nourri par son patron ${ }^{38}$.

14 L'ouvrier, notamment s'il dispose d'un salaire assez élevé, peut donc se permettre, en l'absence d'une véritable discipline au sein de l'atelier, de s'absenter le lundi et de ne travailler que trois, quatre ou cinq jours par semaine. De même, il peut choisir de travailler dans la matinée du dimanche pour augmenter les ressources nécessaires aux dépenses conditionnées par la fête du lundi. L'ouvrier reçoit en général son salaire le samedi, ce qui constitue une autre raison capitale, aux yeux des pourfendeurs de la Saint Lundi, de fêter ce jour-là, simple prolongation alors des libations entamées dès le samedi soir ${ }^{39}$. L'évolution du travail du dimanche à partir des années 1830 rompra cependant cette unité temporelle, ce qui met, au moins partiellement, en cause cette raison. 

l'ancien député d'olivier met en garde les « ouvriers laborieux » contre le fait d'épouser des filles qui font le lundi ${ }^{40}$. Déjà Louis-René Villermé à la fin des années 1830 avait constaté la pratique du chômage du lundi chez certaines ouvrières de Lille et de Reims ${ }^{41}$, alors que Honoré-Antoine Frégier la dénonce ensuite chez les ouvrières parisiennes ${ }^{42}$. La Statistique de l'industrie à Paris relève des métiers féminins, comme celui des fabricantes de masques ou des brodeuses, qui limitent leur temps de travail hebdomadaire à quatre jours par semaine, gagnant respectivement deux à quatre, et quatre à cinq francs par jour ${ }^{43}$. Il s'agit cependant d'un fait exceptionnel car les autres métiers féminins qui chôment le lundi (fileuses et retordeuses de coton, fabricantes de boucles et d'agrafes, de montures de lunettes, ouvrières de bimbeloterie, horlogères...) doivent se contenter de salaires allant de 1,50 à 1,80 franc par jour ${ }^{44}$. Nous ne connaissons pas très bien la situation de ces ouvrières : certaines logent en garni, mais pour le reste, s'agit-il de femmes mariées ou célibataires ? Nous aurions plutôt tendance à conclure à de jeunes ouvrières qui profitent de cette journée pour se rendre aux bals publics ${ }^{45}$, mais des ouvrières mariées ne semblent pas non plus exclues de la fête du lundi ${ }^{46}$. Ce dernier cas, celui de l'épouse qui «suit son mari », pourrait s'expliquer, suivant l'exemple anglais, par le fait que le salaire de la femme mariée sert de salaire d'appoint au ménage ouvrier, auquel on peut donc renoncer périodiquement ${ }^{47}$.

Dans l'ensemble, il s'agit donc chez les adeptes du chômage du lundi durant la première moitié du XIX siècle, d'ouvriers qualifiés, travaillant à la pièce ou à la tâche, dotés d'un grand savoir-faire, aux salaires relativement élevés, logeant en garni, donc plutôt jeunes, célibataires, voire migrants et "nomades ». Cette catégorisation concerne surtout les compagnons qui, en effet, possèdent une longue tradition du chômage du lundi. Des chansons répertoriées par Paul Sébillot montrent à quel point la Saint Lundi fait partie des coutumes des tisserands, cordonniers, sabotiers, chapeliers, tailleurs de pierre, menuisiers, peintres, vitriers, doreurs et autres imprimeurs sous l'Ancien Régime. Au début du XIXe siècle, ce sont donc les compagnons qui servent de vecteur au chômage du lundi, et qui le répandent aussi dans des régions jusque-là plutôt à la marge de sa géographie $^{48}$. Tout en chômant le lundi, ils utilisent ce jour-là pour leurs rites. Ainsi estce très souvent un lundi qu'ils font la "conduite ", c'est-à-dire qu'ils accompagnent un des leurs qui quitte la ville pour reprendre le tour. Ces « conduites » constituent une des raisons majeures des nombreuses rixes entre les diverses sociétés de compagnonnage, car elles sont liées à de fortes libations, sources de chansons provocatrices à l'égard des sociétaires d'autres sociétés ${ }^{49}$. La Saint Lundi s'inscrit donc dans les coutumes des compagnons fortement contestées au XIX $\mathrm{X}^{\mathrm{e}}$ siècle ${ }^{50}$. Ces traditions peuvent à leur tour engendrer de nouvelles coutumes, comme celle des papetiers de Thiers qui oblige les apprentis à payer une forte somme. Les ouvriers consomment ensuite cette somme «en débauches et orgies ", au lieu de se rendre au travail ${ }^{51}$.

Si l'on suivait le discours des élites sur la pratique de la Saint Lundi, le constat d'une journée perdue dans l'ivrognerie s'imposerait. À vrai dire, cette coutume est depuis longtemps étroitement liée à des bacchanales. Le vin fait partie de la Saint Lundi, sans aucun doute, comme il fait partie de toute fête, mais peut-on limiter les usages que font les classes populaires de ce jour-là, à de simples beuveries? Cabarets, guinguettes et bals publics constituent en effet les endroits préférés pour passer le lundi. Ce serait donc dans ces lieux-là qu'on entendrait des «cris inarticulés, des chansons obscènes, des propos licencieux, des provocations ", dans une atmosphère à peine respirable par la fumée des

Revue d'histoire du XIXe siècle, 29 | 2005 
pipes et dominée par l'ennui, selon la description d'un Jules Simon ${ }^{52}$. Si c'est l'ennui qui règne, pourquoi l'ouvrier chômerait-il alors le lundi? En effet, d'autres témoignages fournissent un tableau beaucoup plus joyeux de la manière de passer ce jour-là : selon Paul de Kock, des familles entières, donc pas seulement des ouvriers seuls, se rendent aux barrières pour s'y amuser à la fois les dimanches et les lundis ${ }^{53}$. Les établissements de Belleville sont ouverts le lundi pour un public ouvrier, comme ils le sont le dimanche et le jeudi pour d'autres publics. Certains bals intra-muros à Paris ouvrent également toujours le lundi jusqu'à la fin des années $1870^{54}$. La description faite d'un lundi de deux jeunes femmes dans une guinguette à la barrière de Montparnasse met ainsi en scène des valses et des contredanses, auxquelles s'ajoutent des ripailles assez copieuses, bien arrosées au moins par une partie de leur société. C'est le plaisir que procure le séjour dans cette guinguette, qui compte, et pour lequel l'une d'elles est même prête à mettre son parapluie chez une fripière pour obtenir les 40 sous nécessaires ${ }^{55}$. À Marseille, les « chômeurs » du lundi se retrouvent chez un barbier pour y pratiquer divers jeux et bien manger, alors qu'en été ils se rendent dans les guinguettes du bord de la mer ${ }^{56}$. Les jeux, cartes, quilles etc., font partie du paysage de ce jour, ce que confirme aussi Jules Simon ${ }^{57}$. Ces informations dessinent donc l'image d'un lundi festif et ludique, passé en partie aussi dans un cadre familial. C'est le plaisir qui compte - à Paris comme ailleurs ${ }^{58}$. Eugène Buret se montre d'ailleurs tout à fait conscient de ce fait en dénonçant la recherche de plaisirs chez les ouvriers comme une des raisons pour fêter la Saint Lundi ${ }^{59}$. Mais le lundi peut aussi servir de cadre pour des activités plus culturelles. La fréquentation des théâtres fait ainsi partie des activités dénoncées par les pourfendeurs de la Saint Lundi ${ }^{60}$. De même, lors de l'Exposition universelle de 1855 à Paris, la fréquentation du lundi ressemble presque à celle du dimanche, ce qui incite les organisateurs à transférer la réduction offerte aux ouvriers à ce jour-là ${ }^{61}$ !

Les usages du lundi ne correspondent donc pas tout à fait au tableau noir dépeint par les moralistes, et la sombre description de l'adepte du lundi, ivrogne, violent et immoral, ne reflète absolument pas la vérité - les rapporteurs de la Statistique industrielle sont d'ailleurs obligés de nuancer ce constat eux-mêmes, en montrant des ouvriers aux habitudes « rangées » et " régulières » dans des métiers qui fêtent la Saint Lundi. Ainsi au sujet des serruriers pour meubles, ils écrivent : « Non seulement l'habitude de chômer le lundi est générale dans cette profession, mais encore un grand nombre d'ouvriers ne travaillent que quatre jours par semaine. Beaucoup cependant ont une vie assez régulière et sont laborieux quand ils sont à l'atelier... " ${ }^{62}$.

19 La fête hebdomadaire du lundi, d'une certaine façon, ressemble plutôt au carnaval, dont elle emprunte certains rites et lieux ${ }^{63}$. Et comme le carnaval du peuple, cette fête du lundi, fête bruyante, devient progressivement insupportable aux yeux des élites - les décrets et ordonnances de la police dénoncent alors les « désordres » et les "troubles ", sous forme de rixes dans les faubourgs et à l'intérieur de la ville, commis le lundi, dont les responsables sont ceux qui reviennent, dans la soirée, des guinguettes hors des barrières ${ }^{64}$. La violence, en effet, n'est pas absente de la fête du lundi. La géographie (le Paris actif et populaire, ainsi que les lieux des barrières et des cabarets, comme le quartier des Porcherons et celui de la Courtille) et les temps des violences (le dimanche et surtout le lundi) constatés par Arlette Farge et André Zysberg pour le Paris du XVIII ${ }^{e}$ siècle ${ }^{65}$, restent toujours valables pendant la première moitié du XIX ${ }^{e}$ siècle. Le tourneur en chaises Bédé comme le maçon limousin Léonard connaissent des lundis passés à la barrière où des bagarres se produisent ${ }^{66}$. 
20 À l'origine de ces violences se trouve très souvent la coexistence de divers groupes ethniques, d'Auvergnats et de Limousins par exemple, ainsi que de divers métiers, ou encore celle d'ouvriers et de militaires. La violence compagnonnique choisit également ce jour-là. Sur les 115 rixes entre compagnons que nous avons relevées pour l'époque de la Restauration, à peu près $30 \%$, à savoir 34 , ont lieu un lundi ( 9 sur 21 à Paris), ce qui assure à ce jour la première place pour les rixes, suivi par le dimanche. Ces deux jours chômés dans l'univers des compagnons sont donc aussi les supports temporels préférés pour les violences compagnonniques.

21 La violence fait donc partie des usages du lundi. Elle est toujours, dans cette première moitié du XIXe siècle, intégrée aux activités, aux loisirs et aux modes de vie du monde populaire ${ }^{67}$, mais elle contribue également à rendre la Saint Lundi de plus en plus intolérable aux sensibilités des autres classes de la société.

Certes, le discours contre cette pratique s'inscrit dans une vieille tradition. Les thèmes de ce discours ne varient guère au début du XIX siècle, gardant une forte dimension moralisatrice: il dénonce surtout l'ivrognerie comme corollaire inévitable de cette coutume. La Société de Sobriété d'Amiens considère ainsi la Saint Lundi comme une des causes principales de l'ivrognerie ouvrière ${ }^{68}$. Certains, comme Jules Simon, accusent aussi l'usage du tabac dans ce contexte ${ }^{69}$. Ce rapport avec l'ivrognerie se retrouve dans toutes les diatribes contre le chômage du lundi, entraînant d'autres accusations: celle de la violence, notamment contre les femmes, mettant en péril l'unité familiale. Selon ces descriptions, c'est l'épouse désespérée qui cherche à s'opposer d'abord aux parties de plaisir du mari le lundi, et qui devient ensuite la victime de ses brutalités ${ }^{70}$.

Ce discours est aussi fortement opposé à tout plaisir de l'ouvrier. L'abbé Dreuille dénonce ainsi cette tendance de la classe laborieuse à vouloir s'amuser, car cette recherche de plaisir, notamment le lundi, «se solde toujours en maladies, en regrets et en misère ", et elle est étroitement liée à ces fléaux qui se présentent sous la forme de «libertinage, gourmandise, ivrognerie ${ }^{71}$. La Saint Lundi, aux yeux de ces auteurs moralistes, possède aussi une forte dimension immorale, et assez fréquentes sont les allusions à des lieux de débauche, derrière lesquels se cachent des maisons closes ${ }^{72}$. De toute façon, l'adepte du lundi est aussi celui qui refuse le sacrement du mariage et vit en concubinage ${ }^{73}$.

24 Les dépenses faites dans le cadre d'une journée passée aux barrières et ailleurs constituent un autre grief car elles empêchent toute épargne. Dans un drame, Le Bonhomme Lundi, les auteurs font dire au héros, un certain Griffueil, qui fête la Saint Lundi depuis 40 ans, qu'il aurait pu épargner en y renonçant la coquette somme de 20000 francs, autrement dit qu'il aurait pu s'aménager une "retraite d'officier " ${ }^{74}$. L'abbé Dreuille estime à 400 francs par an les pertes causées par la pratique du lundi : trois francs pour ne pas avoir travaillé, plus cinq francs de dépenses, la somme multipliée par 50 [sic !] semaines ${ }^{75}$. En absence de toute épargne causée par la fête du lundi, le destin de l'ouvrier réside par conséquent dans la pauvreté et la misère. Selon une brochure contre la Saint Lundi, la "mère n'a que des vêtements en lambeaux, les enfants ont cette apparence pâle et chétive que cause une nourriture insuffisante et souvent mauvaise ; l'hiver ils grelottent sous leurs haillons... ${ }^{76}$.

25 Un autre thème récurrent de la plaidoirie anti-lundi réside dans les conséquences néfastes de cette coutume pour l'économie. Le chômage du lundi frappe surtout les manufactures dont le moteur général est une pompe à feu, car il oblige les maîtres, manquant d'ouvriers, à arrêter les machines et ainsi la production ${ }^{77}$. Les fabricants de 
Saint-Quentin, selon Jules Simon, sont même obligés de prévoir le chômage du lundi dans leurs calculs ${ }^{78}$. François Pérennès cite ainsi l'Anarchis des Ateliers qui estime à deux millions de francs les pertes hebdomadaires causées par le chômage du lundi à l'économie de la France ${ }^{79}$. Le statisticien Moreau de Jonnès, en 1851, fixe la somme annuelle perdue pour cause de chômage volontaire, à onze milliards de francs, dépassant ainsi de loin le chiffre indiqué par François Pérennès ${ }^{80}$.

Les griefs traditionnels contre la Saint Lundi sont donc d'ordre moral, social et progressivement économique. Mais depuis les journées de juin 1848, ses pourfendeurs abordent un nouveau thème, celui de l'implication des adeptes de la Saint Lundi dans les émeutes. Ce thème s'inscrit dans une littérature assez riche en faveur du repos du dimanche et contre le chômage du lundi. Cette littérature connaît des pics de parutions d'abord dans les années qui suivent la Révolution de 1848 , et ensuite après la Commune ${ }^{81}$. D'Olivier brosse ainsi, en 1853 le portrait de l'ouvrier chômeur du lundi : « Enfin, dans les désordres sociaux, parmi les faiseurs de barricades, cherchez bien et vous verrez que ce sont les ouvriers qui ne se reposent pas le dimanche et qui font le lundi, qui en sont l'âme, les agents principaux ${ }^{82}$. Pour Antoine d'Indy, le lundi chômé n'est rien d'autre que le temps des "mariannes" et des sociétés secrètes ${ }^{83}$. Mgr de Ségur, dans l'éditorial du premier numéro de l'Ouvrier (11 mai 1861), intitulé «Comment on devient révolutionnaire ", décrit alors l'ouvrier " qui s'insurge contre son patron, lit et commente le Siècle, se plaint du gouvernement, entre dans les sociétés secrètes, acclame la République, fête le lundi, jamais le dimanche et au besoin fait des petites journées comme celles de juin 1848... " ${ }^{84}$. Augustin Cochin, en 1862, oppose le mauvais ouvrier qui fête le lundi, suit les «meneurs », fréquente le cabaret, fait partie des sociétés secrètes et lit le « mauvais journal », à l'ouvrier respectueux du dimanche, du patron, vivant en famille et étant adonné à l'épargne ${ }^{85}$.

Cette tendance à établir un lien direct entre la pratique de la Saint Lundi et la révolte revient donc en force après la Commune. L'abbé Clément brosse ainsi le portrait de Mathieu qui, de retour des événements sanglants de Paris, et «repenti », condamne le lundi, cause principale de ses erreurs ${ }^{86}$. Le lundi, suivant une autre source, devient alors "le laboratoire où se fait l'amalgame d'où sortent les grandes catastrophes " ${ }^{87}$.

Ce lien entre contestation politique et sociale, d'un côté, et la pratique du lundi de l'autre n'est pas tout à fait récent. Déjà en 1823 , un rapport de police de Paris note que le « libéralisme révolutionnaire ne trouve pour appui dans le peuple que les hommes oisifs, abrutis par le vin et la débauche ${ }^{88}$, ce qui signifie les ouvriers qui fêtent le lundi. Cabarets, cafés ainsi que guinguettes constituent en effet des lieux de contestation, comme ce café du boutonnier Gille (il s'agit de Gilbert Désiré Hyppolite), où se réunit la Société des Travailleurs égalitaires tous les dimanches et lundis sous la Monarchie de Juillet ${ }^{89}$. L'importance de la sociabilité de ces lieux le dimanche et le lundi est en effet capitale pour la stratégie des luttes ouvrières, comme l'a souligné Maurice Agulhon ${ }^{90}$. De même, les catégories socioprofessionnelles qui participent aux journées de juin 1848, correspondent entièrement aux critères relevés par la Statistique de l'industrie à Paris pour définir ceux qui chôment le lundi ${ }^{91}$.

La répression politique et policière qui suit les journées de Juin, et qui se renforce avec l'installation de l'Empire autoritaire, de même que les premières mesures prises contre la pratique de la Saint Lundi pendant les années 1850, réduisent cette activité - ce qui peut expliquer le recul de la Saint Lundi durant les années 1850 à Paris. La libéralisation du régime donne ensuite une nouvelle dimension politique à cette pratique. Les «cris 
séditieux » un lundi, dont les auteurs sont des ouvriers, augmentent considérablement à partir de $1866^{92}$. Le tableau que brosse Denis Poulot en 1870 de ses travailleurs, met en évidence des ouvriers «sublimes" et autres «fils de dieu " tout à fait adhérents aux théories socialistes qui fréquentent les réunions politiques ${ }^{93}$. Or celles-ci ont souvent lieu le lundi à Paris ${ }^{94}$. L'enquête sur la situation des classes ouvrières en 1872 montre aussi que ce lien ne se limite pas au seul département de la Seine : les ouvriers de l'industrie de la porcelaine et du textile de Limoges font de la pratique du lundi une question politique, encouragés dans cette attitude par un "parti politique " ${ }^{95}$. Des patrons marseillais se plaignent également des " efforts faits par des meneurs d'amener le chômage du lundi » dans leurs industries ${ }^{96}$. Ce " parti politique », ces «meneurs » ne signifient rien d'autre, aux yeux des observateurs, que l'Association internationale des travailleurs (AIT) qui semble utiliser à la fois le temps et les lieux de la Saint Lundi pour ses propres buts. Dans une enquête menée au début des années 1870 par la police dans le département de la Seine, le commissaire de police du quartier des Archives, quartier où règne la bijouterie et le travail du bronze, métiers pratiquant la Saint Lundi, évoque la loi de 1864 sur les coalitions qui aurait «jeté une perturbation morale chez les ouvriers » en réveillant en eux des "prétentions exorbitantes ", allant jusqu'au point de vouloir les faire "régner dans l'atelier, de faire la loi au Patron... ${ }^{97}$. Avant 1864, selon le même commissaire, il existait l'« harmonie » dans ces industries. Le commissaire du quartier de la Monnaie, haut lieu de l'imprimerie et fief de la Saint Lundi, constate à son tour les mauvais rapports entre ouvriers et patrons, notamment dans les petits ateliers où les ouvriers « ne sont pas assidus au travail [fêtent donc le lundi], se livrent à la boisson, à la paresse, à la lecture des écrits licencieux et révolutionnaires, et se jettent à corps perdu dans les sourdes manœuvres de l'Internationale. Malheureusement, le nombre de ces prétendus ouvriers peut être évalué au deux tiers au moins du nombre des travailleurs dans mon quartier ${ }^{98}$.

Ces exemples montrent donc une politisation croissante de la pratique du lundi depuis les années 1860, qu'il ne faut cependant pas généraliser : dans les régions où la Saint Lundi reste liée au repos dominical, cette évolution n'apparaît pas. Mais pour la plupart des ouvriers des centres industriels, anticléricaux et antibourgeois, l'institution du dimanche est trop entachée de cléricalisme et soumise aux normes de la bourgeoisie. Ils lui préfèrent la fête du lundi, qui, de cette manière, devient aussi la plate-forme temporelle de leurs luttes.

31 Pour les classes dominantes, il s'agit donc de déraciner cette tradition, dorénavant étroitement liée à la contestation et à la révolte. Le premier moyen semble résider dans l'installation obligatoire du repos dominical. Un vaste discours d'origine catholique et philanthropique, prônant les bienfaits du repos dominical pour l'ordre moral et social de la société, tout en condamnant la Saint Lundi pour ses méfaits d'ordre familial et productiviste, lance ses foudres contre cette coutume, avec une intensité variable, plus forte après les révolutions sociales. Mais le travail du dimanche correspond encore trop à la mentalité du laisser-faire imposé par le libéralisme économique, pour qu'une lutte à ce niveau soit envisageable avant la fin du siècle.

Les moralistes cherchent aussi à combattre la Saint Lundi sur un niveau pédagogique : plusieurs pièces de théâtre ${ }^{99}$ ainsi que des nouvelles ou romans édifiants sont censés empêcher les ouvriers de fêter le lundi ${ }^{100}$. Ce thème se retrouve aussi dans des almanachs ${ }^{101}$. Leur contenu est toujours le même : l'ouvrier qui se détache de la religion, qui devient victime de mauvaises influences, qui ne sait pas résister aux tentations, fête enfin le 
lundi, malgré les avertissements de son patron qui le met finalement à la porte de l'atelier. L'ouvrier tourne mal alors en devenant soit criminel soit révolutionnaire - la différence n'est pas grande pour nos moralistes. Le lecteur peut se pencher aussi sur le triste sort d'une famille délaissée par le père et tombée dans la misère, où la prison guette les fils, la prostitution les filles, alors que la vieille mère, gardant un fond de foi, va mourir de chagrin. Des variations de ce thème existent, mais la Saint Lundi comme racine de tous les maux qui frappent la société reste la grande constante.

Ces mesures pédagogiques n'obtiennent aucun résultat. La disparition partielle du lundi chômé dans certaines industries avant 1880, notamment dans celles qui se servent de la force des machines, est surtout la conséquence d'une politique répressive des patrons, notamment depuis 1848 et renforcée après 1871, appliquant le principe de la carotte et du bâton. La ville de Sedan où les patrons du textile se sont concertés pour renvoyer tout ouvrier fêtant le lundi, tout en établissant un système paternaliste de protection sociale, devient le symbole de cette politique ${ }^{102}$. Des centres industriels alsaciens (Mulhouse, Guebwiller...), normands (Elbeuf) et méridionaux (Tarare) voient naître la même politique de la part des industriels. Dans les mines d'Anzin, les responsables empêchent ainsi les chômeurs du lundi de descendre dans les fosses ${ }^{103}$. À Lille, à Cambrai, ou encore à Bayonne, ils sont exclus de toute aide par les bureaux de bienfaisance ou par les sociétés de secours mutuel ${ }^{104}$.

La politique de la carotte en revanche réside dans la distribution de primes à ceux qui boycottent le lundi : les filateurs du Maine-et-Loire leur accordent une prime de $10 \%{ }^{105}$, d'autres, comme le teinturier Guéroult à Rouen, donnent 25 centimes de plus pour chaque jour si l'ouvrier ne chôme pas ${ }^{106}$. Certains patrons, comme l'entrepreneur en peinture parisien Leclaire et le joaillier Rouvenat, essaient avec un certain succès la participation de leurs ouvriers ${ }^{107}$. Les patrons du Nord, quant à eux, accordent à leurs ouvriers la sortie à quatre heures de l'après-midi pour empêcher «en amicale» le chômage du lundi ${ }^{108}$. Sans aller aussi loin, les filateurs du Laonnais offrent le lundi un verre d'eau-de-vie à leurs ouvriers ${ }^{109}$.

Payer les ouvriers un autre jour que le samedi, voire le dimanche matin, constitue un autre moyen d'empêcher la fête du lundi : des industriels de Roubaix, à l'instar de MotteBossuet, choisissent le mercredi comme jour de paye ${ }^{110}$, alors que leurs collègues de Nantes préfèrent le vendredi pour que le marché du samedi absorbe la somme normalement consacrée aux dépenses du lundi ${ }^{111}$. D'autres industriels encore, au lieu de verser la paye par semaine ou par quinzaine, ne le font que mensuellement, acceptant à cette occasion un ou deux jours de chômage volontaire de leurs ouvriers ${ }^{112}$.

Certains industriels inscrivent aussi la lutte contre la Saint Lundi dans leur politique paternaliste, comme la Société industrielle de Mulhouse ${ }^{113}$ créant un cercle d'ouvriers pour empêcher la visite du cabaret, et donc le chômage du lundi. Certaines associations à caractère ludique contribuent en effet à faire reculer le chômage du lundi, comme les sociétés chorales chez les typographes parisiens ${ }^{114}$.

37 Rares sont en revanche ceux qui réclament la voie législative pour supprimer définitivement le chômage du lundi : la demande du comte de la Tour lors de la discussion du budget en 1869 reste sans écho ${ }^{115}$. Les voix « anti-lundi » insistent beaucoup plus, d'un côté, sur l'action des patrons, et, de l'autre côté, sur l'intervention morale des femmes : elles leur accordent un rôle majeur pour rétablir le repos dominical et, par conséquent, déraciner la Saint Lundi ${ }^{116}$. C'est aussi sous cet angle qu'il faut regarder la tentative d'importer la semaine anglaise, c'est-à-dire le samedi après-midi libre, dans les 
entreprises pour combattre le chômage du lundi. Cette demi-journée est censée permettre aux femmes ouvrières d'effectuer les tâches ménagères pour qu'elles soient ensuite entièrement disponibles le dimanche - de cette manière, l'ouvrier ne serait pas tenté de travailler ce jour-là, mais le lundi ${ }^{117}$.

Cependant, toutes ces tentatives n'obtiennent qu'un succès limité avant 1880. La Saint Lundi reste vivante dans les ateliers qui gardent le caractère traditionnel du travail industriel, s'appuyant sur le savoir-faire de l'ouvrier - que l'on pense au bel exemple de l'atelier de Denis Poulot. Les mesures disciplinaires ne montrent que peu d'effets car les ouvriers possèdent dans ces structures la possibilité de changer de patron selon leurs souhaits, alors que les patrons dépendent de leur maîtrise, notamment à une époque qui se caractérise par une pénurie de main-d'œuvre - ce qui explique que de nombreux patrons «ferment les yeux " ${ }^{118}$ car «les bras manquent» ${ }^{119}$. L'introduction de mesures répressives contre le chômage du lundi rencontre en outre une très grande résistance $d u$ côté des ouvriers ${ }^{120}$. Chez Holden à Reims, les ouvriers réagissent par une grève contre l'introduction de mesures disciplinaires les empêchant de fêter le lundi ${ }^{121}$. Dans l'industrie textile de Tourcoing, tout un système d'amendes échoue ainsi face à l'opposition des travailleurs ${ }^{122}$. Il s'agit pour ces ouvriers de défendre un élément capital de leur identité, menacée par le processus lent, mais inévitable, de leur prolétarisation.

Cependant, la pratique de la Saint Lundi va se marginaliser progressivement. L'enquête ministérielle menée en 1893 confirme l'image d'une tradition en plein recul ${ }^{123}$. Même à Paris, son ancien fief, le chômage du lundi ne se trouve plus que dans le bâtiment au début des années $1890^{124}$. La politique répressive contre la Saint Lundi lancée après la Commune a certainement contribué à ce recul. Mais d'autres facteurs, d'ordre structurel et mental, entrent également en jeu.

La crise économique fait apparaître le spectre du chômage involontaire, toujours opposé à la fête du lundi ${ }^{125}$. La même crise économique constitue aussi un point de passage vers une mécanisation et une motorisation générales de l'industrie, incompatibles avec l'autodétermination du temps ouvrier. À cela s'ajoute une prolétarisation progressive (mais incomplète) de la classe ouvrière qui la prive également de toute autonomie et l'enferme dans les « bagnes » des usines, régis par une discipline impitoyable.

41 Cependant, toutes ces mesures disciplinaires et mutations structurelles ne suffisent pas à expliquer la marginalisation progressive de la Saint Lundi, si l'on ne prend pas en considération le changement de la perception de cette dernière chez les ouvriers euxmêmes durant les deux dernières décennies du siècle. Des voix critiques au sujet de la Saint Lundi émergent des milieux populaires depuis des années 1840. Le vertueux compagnon Agricol Perdiguier en 1841, le journal buchézien L'Atelier en 1844, le papetier Mollet en 1867 condamnent déjà la pratique de la Saint Lundi pour des raisons morales ${ }^{126}$. Mais c'est surtout la campagne que le mouvement ouvrier mène depuis les années 1880 contre l'alcoolisme des travailleurs qui condamne également la fête du lundi. Sa recherche d'une nouvelle respectabilité met en exergue l'ouvrier bon père de famille, et l'oppose à l'adepte de la Saint Lundi. Dans ce contexte, la classe ouvrière adopte ellemême l'imagerie hideuse de la Saint Lundi, conçue depuis des décennies par les classes dominantes. Une pièce de théâtre de 1885 met ainsi en opposition le bon ouvrier, qui se repose le dimanche, se promène ce jour-là avec sa famille, ne fête pas le lundi et accepte la valeur du travail, tout en aspirant à l'émancipation de la classe ouvrière, à l'ouvrier ivrogne, adepte du chômage du lundi, qui tient un discours révolutionnaire, certes, mais complètement incohérent et discrédité par son propre comportement immoral ${ }^{127}$. Autre 
témoignage de cette nouvelle attitude ouvrière, une feuille syndicale, L'Ouvrier de l'Est, en 1898: "Que l'habitude de faire lundi soit proscrite de nos mœurs ouvrières et tout le monde sera content. C'est la grâce que je vous souhaite " ${ }^{128}$.

Dans ces mêmes années, à partir de 1880 , on constate en revanche le retour du repos dominical dans les habitudes ouvrières. Les lois scolaires imposent un rythme hebdomadaire centré autour du dimanche, et elles donnent ainsi une nouvelle valeur à la vie familiale. Le dimanche constitue également la plate-forme temporelle pour les loisirs dans une société caractérisée par la nouvelle culture des masses, de même pour des activités civiques dans une société profitant des libertés républicaines. Le mouvement ouvrier fait ainsi de la conquête du repos dominical même un de ses objectifs ${ }^{129}$, au détriment de la fête du lundi.

Le XIX ${ }^{e}$ siècle représente donc à la fois l'apogée et le déclin de la Saint Lundi. Cette tradition, véhiculée par les coutumes compagnonniques, réussit à s'intégrer dans la première vague d'industrialisation en France, et à s'étendre même dans ce cadre. La Saint Lundi obtient même son autonomie dans ce contexte, en se décrochant du repos du dimanche, et en s'opposant désormais à celui-ci. Un premier recul, causé par la répression qui suit les journées de juin 1848, intervient pendant les années 1850, avant que les années 1860 ne connaissent un nouvel essor de la Saint Lundi, cette fois-ci étroitement lié à la politisation de ses usages. Après le double choc de la défaite de 1871 et de la Commune, il s'agit donc pour la bourgeoisie de supprimer définitivement cette coutume du monde ouvrier, dont la pratique se trouve contraire à la fois aux conceptions productivistes de la bourgeoisie industrielle et aux conceptions conservatrices des classes dominantes. Dénigrer le révolutionnaire de 1848 et de 1871 comme un simple ivrogne, chômeur du lundi, asocial et irresponsable, fait donc partie d'une stratégie développée dans un discours qui ne reflète absolument pas la réalité, à part l'adéquation entre révolutionnaire et adepte du lundi. Ce dernier représente surtout un ouvrier fier de son autonomie dont la fête du lundi constitue le symbole.

La tentative de la bourgeoisie de supprimer la Saint Lundi et de synchroniser le temps social ne sera couronnée de succès qu'à partir du moment où la classe ouvrière acceptera elle-même cette synchronisation pour s'adapter aux nouvelles conditions sociales et culturelles de la société de la fin du XIX ${ }^{e}$ siècle. Toutefois, avec la Saint Lundi disparaît définitivement la notion de fête de la vie ouvrière et populaire - la classe laborieuse va dorénavant adopter les normes imposées par la bourgeoisie, pour les utiliser en partie à ses propres fins...

\section{NOTES}

1.. Pour la France : Georges DUVEAU, La vie ouvrière en France sous le Second Empire, Paris, Éditions Gallimard, 1946, pp. 243-248 ; Michelle PERROT, Les ouvriers en grève. France, 
1871-1890, Lille, Service de reproduction des thèses, Université de Lille III, 1975, 2 tomes, tome 1, pp. 109-110; Alain CAILLAUX, Vie et mort de la Saint Lundi au dix-neuvième siècle, mémoire de maîtrise en histoire contemporaine, Université de Paris VII, 1977 ; Jerry KAPLOW, « La fin de la Saint Lundi. Étude sur le Paris ouvrier au XIX ${ }^{\mathrm{e}}$ siècle », dans Temps libre, $n^{\circ} 2,1981$, pp. 107-118. Pour l'Europe : Edward P. THOMPSON, « Time, WorkDiscipline, and Industrial Capitalism », dans Past and Present, $n^{\circ} 38$, décembre 1967, pp. 56-97 ; Douglas A. REID, « The Decline of Saint Monday », dans Past and Present, $\mathrm{n}^{\circ}$ 71, mai 1976, pp. 76-101; Jürgen REULECKE, « Vom blauen Montag zum Arbeiterurlaub. Vorgeschichte und Entstehung des Erholungsurlaubs für Arbeiter vor dem Ersten Weltkrieg ", dans Archiv für Sozialgeschichte, n 16, 1976, pp. 205-248 ; Josef EHMER, « Rote Fahnen - Blauer Montag. Soziale Bedingungen von Aktions- und Organisationsformen der frühen Wiener Arbeiterbewegung ", dans Detlev PULS [dir.], Wahrnehmungsformen und Protestverhalten. Studien zur Lage der Unterschichten im 18. und 19. Jahrhundert, Francfort, Éditions Suhrkamp, 1979, pp. 143-174 ; Douglas A. REID, « Der Kampf gegen den Blauen Montag 1766 bis 1876 », dans idem, pp. 265-296. ; Lars MAGNUSSON, « Protoindustrialisation, culture et tavernes en Suède (1800-1850) », dans Annales. Économies, sociétés, civilisations, tome $45, \mathrm{n}^{\circ} 1$, janvier-février 1990, pp. 21-36.

2.. Dimanche Catholique, $\mathrm{n}^{\circ} 1$ 1, 1875/6, pp. 286-287 ; Jean-Joseph HUGUET, Terribles punitions des profanateurs scandaleux du dimanche, Bar-le-Duc, Librairie Philipona, 1880, p. VI. Pour les origines médiévales, voir Roger PINON, « Le lundi perdu. Une page de folklore social », dans Bulletin FEB, $1^{\mathrm{er}}$ décembre 1973, pp. 2-17. Je profite de cette occasion pour exprimer tous mes remerciements à l'équipe du Musée de Compagnonnage de Tours pour son soutien.

3. Léon NARDIN et Julien MAUVEAUX, Histoire des corporations d'arts et métiers des Ville et Conté de Montbéliard et des seigneuries en dépendant, Paris, Librairie ancienne Honoré Champion, 1910, 2 tomes, tome 1, p. 59 ; pp. 163-164 ; p. 310.

4.. Alfred FRANKLIN, Dictionnaire historique des arts, métiers et professions exercés dans Paris depuis le treizième siècle, Paris, H. Welter, 1906, p. 263.

5. Maurice GARDEN, Lyon et les Lyonnais au XVIII ${ }^{e}$ siècle, Paris, Les Belles Lettres, 1970, p. 569.

6.. Paul SEBILLOT, Légendes et curiosités des métiers, Paris, Éditions Flammarion, 1981, passim.

7.. Jacques-Antoine DULAURE, Nouvelles descriptions des curiosités de Paris, Paris, Lejay, 1785 , p. 282.

8. Thomas BRENNAN, Public Drinking and Popular Culture in Eighteenth Century Paris, Princeton (New Jersey), Princeton University Press, 1988, p. 170.

9.. Arch. nat. (Archives nationales), AD XVIII C 298. SAINT-AUBIN, Rapport sur les fêtes décadaires, an III, p. 4.

10.. Arch. nat., F7 3787.

11.. Guillaume de BERTIER de SAUVIGNY, La Restauration, dans Nouvelle Histoire de Paris, tome 7, Paris, Association pour la publication d'une histoire de Paris, 1977, p. 235.

12.. Adolphe BLANQUI, Des classes ouvrières en France pendant l'année 1848, Paris, Librairie Pagnerre/Paulin et Cie/Librairie Firmin Didot frères, 1849, pp. 31-33.

13. Georges et Hubert BOURGIN, Le régime de l'industrie en France de 1814 à 1830. Recueil de textes, Société d'histoire contemporaine, Paris, Picard, 1912-1941, 3 tomes, tome 1, p. 10.

14.. Idem, tome 3, p. 214.

15.. Victor GELU, Marseille au XIX ${ }^{e}$ siècle, Paris, Éditions Plon, 1971, p. 143. 
16.. John M. MERRIMAN, The Margins of City Life. Explorations on the French Urban Frontier, 1815-1851, New York, Oxford University Press, 1991, traduction française Aux marges de la ville. Faubourgs et banlieues en France, 1815-1870, Collection « L'Univers historique », Paris, Éditions du Seuil, 1994, p. 206.

17.. Louis-René VILLERMÉ, Tableau de l'état physique et moral des ouvriers employés dans les manufactures de coton, de laine et de soie, Paris, Librairie Jules Renouard et $C^{\mathrm{ie}}, 1840,2$ tomes, tome 1, p. 323.

18.. Armand AUDIGANNE, Les populations ouvrières de la France dans le mouvement social du XIXe siècle, Paris, Capelle, 1854, 2 tomes, tome 1, p. 239.

19.. Idem, tome 2, p. 89.

20.. De toute façon, le chômage du lundi n'est pas inconnu dans ces villes comme le montre l'exemple des imprimeurs lyonnais au XVIII ${ }^{\mathrm{e}}$ siècle.

21.. Chambre de commerce de Paris, Statistique de l'industrie de Paris, résultant de l'enquête faite par la Chambre de Commerce pour l'année 1860, Paris, Chambre de commerce, 1860, p. XLIV.

22.. Pierre VINCARD, Les ouvriers de Paris. Études de mœurs, Paris, Michel, 1851, pp. 120-121. 23. Arch. nat., C 3023, Indre-et-Loire. Godeau-Labbé, fabricants-tanneurs à ChâteauRenault. Chambre de commerce de Tours.

24.. Au sujet du travail du dimanche, voir notre ouvrage L'histoire du Dimanche, de 1700 à nos jours, Paris, Éditions de l'Atelier, 1997, pp. 181-207.

25.. Théodore-Henri BARRAU, Conseils aux ouvriers sur les moyens d'améliorer leur condition, Paris, Librairie Hachette,1850, p. 70.

26. Arch. nat., C 3018-3023. Enquête sur la situation des classes ouvrières (1872-1875).

27.. Saint-Lundi. Litanies de Saint-Lundi, Metz, Imprimerie de Debour, s.d.

28.. Victor GELU, ouv. cité, p. 143.

29. L'Affaire Noiret, présentée par Jean-Pierre Chaline, Rouen, Société de l'histoire de Normandie, 1986, p. 101.

30.. Paul DELSALLE, La Brouette et la Navette. Tisserands, paysans et fabricants dans la région de Roubaix et de Tourcoing (Ferrain, Mélanois, Pévèle), 1800-1848, Westhoek, Éditions des Beffrois, 1985, pp. 136-137.

31.. Chambre de commerce de Paris, Statistique de l'industrie à Paris, Paris, Chambre de Commerce,1851, p. 198.

32.. Louis-René VILLERMÉ, ouv. cité, tome 2, p. 66.

33. Louis REYBAUD, Études sur le Régime des manufactures. Condition des ouvriers en soie, Paris, Éditions Michel Lévy frères, 1859, p. 27 ; Jules CLAYE, De la question d'augmentation du salaire des compositeurs typographes. Lettre à M. le rédacteur en chef du journal « Le Courrier du dimanche ", Paris, Imprimerie de Jules Claye, 1861, pp. 21-22.

34.. Annales de la Charité, $\mathrm{n}^{\circ} 10,1854$, p. 340.

35.. Chambre de commerce de Paris, Statistique..., 1851, ouv. cité, p. 764.

36. L'Affaire Noiret..., ouv. cité, p. 101. Ces ouvriers sont payés à la pièce, avec un salaire de six à neuf francs par semaine. Idem, p. 72.

37.. Chambre de Commerce de Paris, Statistique..., 1851, ouv. cité, p. 812.

38. Armand AUDIGANNE, ouv. cité, tome 1, p. 239.

39.. Louis-René VILLERMÉ, ouv. cité, tome 2, p. 67 ; Aperçu sur la condition des classes ouvrières et critique de l'ouvrage de M. Buret, sur la misère des classes laborieuses, par le $\mathrm{p}$ [rin]ce D. S., Paris, Bureau, 1844, p. 28 ; Jules SIMON, L'ouvrière, Paris, Librairie Hachette 1861, p. 133. 
40.. Albert-Joseph-August d'OLIVIER de PEZET, Le repos du dimanche, 1853, texte reproduit dans L'Observateur du Dimanche, $\mathrm{n}^{\circ}$ 4, 1857, p. 240.

41.. Louis-René VILLERMÉ, ouv. cité, tome 1, p. 84 ; p. 106.

42.. Honoré-Antoine FREGIER, Des classes dangereuses de la société dans les grandes villes et des moyens de les rendre meilleures, Paris, chez J.-B. Baillière, 1840, 2 tomes, tome 1, p. 86 ;

p. 100.

43.. Chambre de commerce de Paris, Statistique..., 1851, ouv. cité, p. 764 ; p. 332.

44.. Idem, p. 367 ; p. 572 ; p. 764 ; p. 812 ; p. 820.

45.. Voir la description d'un lundi à la barrière, dans Mœurs parisiennes. Un lundi à la barrière du Mont-Parnasse, Paris, Imprimerie Herban, 1831 : il s'agit de deux jeunes ouvrières qui vont manger et danser à la barrière, pour y rencontrer leurs deux cavaliers. 46.. Jean-Baptiste PUJOULX, Paris à la fin du XVIII e siècle, Paris, B. Mathé, an IX-1801, p. 58 ; Honoré-Antoine FREGIER, ouv. cité, tome 1, p. 86.

47. Douglas A. REID, « Der Kampf gegen den Blauen Montag », art. cité, p. 279. L'auteur cherche ainsi à expliquer le chômage du lundi des femmes ouvrières de Birmingham. 48. Voir l'exemple de Mazamet, décrit par Armand Audiganne. Louis-René Villermé, quant à lui, constate dans les fabriques alsaciennes, que ce sont souvent uniquement les compagnons qui se reposent le lundi. Louis-René VILLERMÉ, ouv. cité, tome 1, p. 64. 49.. GOSSET, père des compagnons forgerons, « Projet tendant à régénérer le compagnonnage sur le tour de France, soumis à tous les ouvriers », 1842, dans Alain FAURE et Jacques RANCIERE [dir.], La Parole ouvrière, 1830-1851, Paris, Éditions 10/18, 1976. 50. Théodore FIX, Observations sur l'état des classes ouvrières, Paris, Guillaumin, 1846, p. 84 ; p. 87.

51.. Lettre de la Chambre consultative des manufactures, arts et métiers de Thiers au ministre de l'Intérieur, 9 mars 1815, citée dans Georges et Hubert BOURGIN, ouv. cité, tome 1, p. 10.

52.. Jules SIMON, ouv. cité, p. 126.

53.. Paul de KOCK, La Grande Ville. Nouveau tableau de Paris, comique, critique et philosophique, Paris, Marescq, 1844, 2 tomes, tome 1, p. 234.

54.. Arch. préf. police Paris (Archives de la préfecture de police de Paris), Db 60. Préfecture de police. Service à fournir par la garde Républicaine dans les établissements publics ci-après. 11 novembre 1871. Ces bals se concentrent sur les $3^{\mathrm{e}}, 4^{\mathrm{e}}, 12^{\mathrm{e}}$ et $15^{\mathrm{e}}$ arrondissements. Un « Etat nominatif des Bals Musettes » (Arch. préf. police Paris, Db 60) du 24 juin 1879 indique toujours le lundi comme jour d'ouverture.

55.. Mœurs parisiennes..., ouv. cité.

56.. Victor GELU, ouv. cité, pp. 143-144.

57.. Jules SIMON, ouv. cité, p. 126.

58. Simone Delattre constate que Paris constitue aussi une ville de plaisirs pour les ouvriers à forts salaires, et pas uniquement pour la « classe de loisir » (Simone DELATTRE, Les douze heures noires. La nuit à Paris au XIX ${ }^{e}$ siècle, Paris, Éditions Albin Michel, 2000, p. 184). Ce caractère de plaisir que procure la possibilité de chômer le lundi, se retrouve aussi dans les villes de province, toutes dotées des mêmes établissements de plaisirs, comme les guinguettes...

59.. Eugène BURET, De la misère des classes laborieuses en Angleterre et en France, Paris, Paulin, 1840, 2 tomes, tome 1, p. 253.

60.. Antoine d'INDY, « De l'influence du dimanche dans la famille », dans Observateur du Dimanche, $\mathrm{n}^{\circ} 3$, 1856, p. 80 ; Le Lundi de l'ouvrier, par un chef d'atelier, Annecy, C. Burdet, 1861, p. 52. 
61.. Observateur du Dimanche, $\mathrm{n}^{\circ} 3,1856, \mathrm{p} .8$.

62.. Chambre de commerce de Paris, Statistique..., 1851, ouv. cité, p. 456. Douglas A. REID constate, quant aux usages de la Saint Lundi à Birmingham, une évolution des activités, situées d'abord dans les tavernes, ou autour des « sports » comme la boxe et les combats d'animaux, vers des activités plus culturelles (théâtre, visites du jardin botanique, excursions), associatives (réunions des sociétés de secours mutuel) et politiques (réunions des Chartistes etc.), donc le recul d'une culture populaire en faveur d'activités plus conformes aux exigences de la bourgeoisie. Douglas A. REID, « Der Kampf gegen den Blauen Montag... ", art. cité, passim. Nous n'excluons pas une telle évolution pour une partie des ouvriers français, mais l'état de sources nous interdit une confirmation définitive.

63.. Voir, à ce sujet, Alain FAURE, Paris Carême-prenant. Du Carnaval à Paris au XIX ${ }^{e}$ siècle, Paris, Librairie Hachette, 1978, 176 p.

64.. Arch. préf. police Paris, Db 60. Préfecture de Police. Circulaire aux maires des communes rurales du département de la Seine, 20 octobre 1818.

65.. Arlette FARGE et André ZYSBERG, « Les théâtres de la violence à Paris au XVIII ${ }^{\mathrm{e}}$ siècle ", dans Annales. Économies, sociétés, civilisations, tome $34, \mathrm{n}^{\circ} 5$, septembre-octobre 1979, p. 987 ; p. 989.

66.. Rémi GOSSEZ, Un ouvrier en 1820. Manuscrit inédit de Jacques-Etienne Bédé, Paris, Presses universitaires de France, 1984, pp. 299-301 ; Martin NADAUD, Mémoires de Léonard, ancien garçon maçon, Paris, Éditions La Découverte, 1998, p. 133.

67.. Arlette FARGE et André ZYSBERG, art. cité, p. 989.

68.. L.-A. LABOURT, Société de sobriété d'Amiens, premier concours ouvert sur les moyens de combattre l'intempérance en France. Essai sur l'intempérance des classes laborieuses et l'établissement en France des sociétés de sobriété, Amiens, Imprimerie R. Machart, 1837, p. 20. 69.. Jules SIMON, ouv. cité, p. 126. L'usage du tabac peut revêtir une signification politique, au point qu'en Prusse et en Autriche, fumer dans les espaces publics est interdit, car signe d'appartenance à l'opposition et aux idées démocrates. Les dénonciateurs du lundi en France n'auraient-ils pas aussi cette image en tête? 70.. À titre d'exemple : abbé Jacques-Isidore MULLOIS, Le dimanche aux classes élevées de la société ou Manuel de l'œuvre du dimanche, Paris, Périsse frères, 1854, pp. 10-12.

71. Discours prononcés aux réunions des ouvriers de la Société de Saint-François-Xavier à Paris et en province par M. l'abbé François-Auguste Dreuille, recueillis et publiés par l'abbé Faudet, curé de Saint-Roch, Paris, au Presbytère de Saint-Roch, 1861, pp. 265-267.

72.. À titre d'exemple : Observateur du Dimanche, $\mathrm{n}^{\circ} 1,1854, \mathrm{p} .11$.

73.. Théodore FIX, ouv. cité, p. 75. La lutte de l'Église contre le concubinage au sein de la classe ouvrière s'effectue sous forme de l'Euvre de Saint François-Regis, destinée à combattre cette forme inacceptable de vie commune, source d'autres vices. Annales de la Charité, $\mathrm{n}^{\circ} 10,1854$, p. 105.

74.. C. LERMITE et A. NETTER, Le Bonhomme Lundi, drame en cinq actes, Paris, Éditions Michel Lévy frères, 1858, p. 9.

75.. Discours prononcés..., ouv. cité, p. 72.

76.. Saint-Lundi, s.l., s.d. [avant 1875], p. 7. Cette brochure se trouve à la Staatsbibliothek de Berlin.

77.. Aperçu..., ouv. cité, p. 29.

78.. Jules SIMON, ouv. cité, p. 136. 
79.. François PÉRENNÈS, De l'Observation du dimanche considérée sous les rapports de l'hygiène publique, de la morale, des relations de famille et de cité, Paris/Besançon, Outhenin-Chalandre fils, 1839 , p. 142.

80.. Cité par Joseph LEFORT, Du Repos hebdomadaire au point de vue de la morale, de la culture intellectuelle et du progrès de l'industrie, Paris, Guillaumin, 1874, p. 133.

81.. Voir le recensement fait d'ouvrages de source catholique concernant le repos du dimanche, ce qui implique la condamnation du lundi de l'ouvrier, dans Robert BECK, ouv. cité, p. 262, note 29.

82.. Albert-Joseph-August d'OLIVIER de PEZET, art. cité, p. 240.

83.. Antoine d'INDY, art. cité, p. 80.

84.. Cité par Pierre PIERRARD, Enfants et jeunes ouvriers en France (XIX ${ }^{e}-X X^{e}$ siècles), Paris, Éditions Ouvrières, 1987, p. 187.

85.. Augustin COCHIN, De la condition des ouvriers français d'après les derniers travaux, Paris, C. Douniol, 1862, p. 37.

86.. Abbé Gilbert CLÉMENT, L'ouvrier \& le dimanche ou Entretiens familiers chez le menuisier Philippe, Paris, Dillet, 1871, p. 9. Pour son interlocuteur, également un ancien adepte du chômage du lundi, celui-ci signifie même «l'exploitation de l'homme par l'homme ", ibidem.

87.. Dimanche ou lundi, Arras, Imprimerie Sede \& Cie, 1875.

88.. Georges et Hubert BOURGIN, ouv. cité, tome 2, p. 180.

89.. Article « Gille ", dans Claude PENNETIER [dir.], Dictionnaire biographique du mouvement ouvrier français [CD-ROM], Paris, Éditions de l'Atelier, 1997.

90.. Maurice AGULHON, « Classe ouvrière et sociabilité avant 1848 », dans Histoire vagabonde, 2 tomes, tome 1, Ethnologie et politique dans la France contemporaine, Paris, Éditions Gallimard, 1988, pp. 60-97.

91.. Voir Maurice AGULHON, Les Quarante-huitards, Paris, Éditions Gallimard, 1992 (1 ${ }^{\text {ère }}$ édition 1975), p. 32.

92.. Suivant un sondage fait dans la série BB 18 des Archives nationales.

93.. Denis POULOT, Question sociale. Le sublime, ou le travailleur comme il est en 1870 et ce qu'il peut être, Paris, Lacroix, Verbœckhoven et $\mathrm{C}^{\mathrm{e}}, 1872$, passim.

94.. Alain DALOTEL, Alain FAURE et Jean-Claude FREIERMUTH, Aux origines de la Commune. Le mouvement des réunions politiques à Paris, 1868 - 1870, Paris, Éditions Maspero, 1980, p. 67. 95.. Arch.nat., C 3023. Haute-Vienne, Ardant, porcelaines, Limoges ; Duboucheron aîné, tissage, Limoges.

96.. Arch. nat., C 3021. Bouches-du-Rhône, Rostand, huilerie, Marseille.

97.. Arch. préf. police Paris, BA 400. Conditions du travail en France, Commissariat de police du quartier des Archives.

98.. Arch. préf. police Paris, BA 400. Conditions du travail en France, Commissariat de police du quartier de la Monnaie, $6^{\mathrm{e}}$ arrondissement. On constate en effet chez les bronziers et les typographes un « taux de syndicalisation » assez élevé avant 1871, voir Jacques ROUGERIE, Paris libre, 1871, Paris, Éditions du Seuil, 2004 (1 ère édition 1971), p. 23. 99.. La Saint-Lundi ronde chantée au théâtre des Funambules, Paris, Imprimerie d'E. Vert, 1862 ; Florentin LEFILS, Le [sic] Saint-lundi, vaudeville en un acte, Abbeville, J. Gamain, 1864. 100.. Le Bonhomme Lundi, ouv. cité ; Les Observateurs du Dimanche et les Faiseurs de Lundi, Paris, Dépôt central des publications relatives au Repos du Dimanche, 1855 ; abbé Nazaire ARNAUD, Nouvelles morales des faubourgs, Paris, C. Douniol, 1855 ; Le lundi de l'ouvrier par un chef d'atelier, Annecy, C. Burdet, 1861 ; abbé Gilbert CLÉMENT, ouv. cité. De nombreuses 
histoires de ce genre se trouvent aussi dans L'Observateur du Dimanche (1854-1867) et Le Dimanche Catholique (à partir de 1875).

101.. Almanach pour l'année 1854, Paris, Railly, pp. 31-39.

102.. Théodore FIX, ouv. cité, p. 83.

103.. Joseph LEFORT, Études sur la moralisation et le bien-être des classes ouvrières : intempérance et misère, Paris, Guillaumin, 1875, p. 317.

104.. Arch. nat., C 3023. Basses-Pyrénées, Chambre de commerce de Bayonne ; Joseph LEFORT, Du repos hebdomadaire..., ouv. cité, p. 94 ; Joseph LEFORT, Études sur la moralisation..., ouv. cité, p. 315.

105.. Arch. nat., C 3020. Maine-et-Loire, Filateurs du Maine-et-Loire.

106.. Arch. nat, C 3020, Seine-Inférieure.

107.. Jean-Edme LECLAIRE, Des améliorations qu'il serait possible d'apporter dans le sort des ouvriers peintres en bâtiments, suivies des règlements d'administration et de réparation des bénéfices que produit le travail, Paris, Imprimerie Mme Veuve Bouchard-Huzard, 1843 ; Maison L. Rouvenat, Inauguration des nouveaux ateliers de joaillerie \& de bijouterie, 27 septembre 1866, Paris, Imprimerie Renou et Maulde, 1867.

108. Arch. nat., C 3018. Pas-de-Calais, Hubert, tanneur-mégissier, Boulogne-sur-Mer. 109.. Arch. nat., C 3019. Aisne, Demeaux, filateur à Chavusse (arrondissement de Laon).

110.. Jules SIMON, ouv. cité, p. 135.

111.. Arch. nat., C 3020. Loire-Inférieure, Conseil d'hygiène de Nantes.

112.. Arch. nat., C 3023. Puy-de-Dôme, Directeur de la Société des Mines de Brassac.

113.. Jules SIEGFRIED, Les cercles d'ouvriers. Conférence faite au Havre le 29 novembre 1874, Le Havre, Imprimerie F. Santallier \& Cie, 1874.

114.. Julien HAYEM, Le repos hebdomadaire, Paris, Didier, 1873, p. 270.

115.. Joseph LEFORT, Du Repos hebdomadaire..., ouv. cité, p. 45.

116.. Robert BECK, ouv. cité, pp. 278-280.

117.. Dimanche Catholique, $\mathrm{n}^{\circ} 2$, juillet 1877, pp. 362-366.

118.. Arch. nat., C 3018. Haut-Rhin, Michel Page, constructeur mécanicien, Valdoie près Belfort.

119.. Arch. nat., C 3018. Pas-de-Calais, Chambre de commerce de Saint-Omer.

120.. « La plus grande résistance quand ils [les ingénieurs] veulent modifier leurs habitudes » écrit à ce sujet le préfet de la Haute-Saône en 1872 (Arch. nat., C 3018).

121.. Georges DUVEAU, ouv. cité, pp. 245-246.

122.. Joseph LEFORT, Études sur la moralisation..., ouv. cité, p. 316.

123. Ministère du Commerce. Office du Travail, Enquête sur les salaires et la durée du travail dans l'industrie française (grande et moyenne industrie), Paris, Imprimerie nationale, 1893-1897, 5 tomes, tome 3, pp. 550-624.

124.. Repos du Dimanche, $\mathrm{n}^{\circ} 3,1893, \mathrm{p} .157$.

125.. Lors de l'enquête parlementaire de 1872-1875, certains rapporteurs expliquent l'absence de la Saint Lundi dans leurs branches par l'existence de chômages périodiques. 126.. Agricol PERDIGUIER, Le livre du compagnonnage, Marseille, Laffitte Reprints, 1978 ( 1 ère édition 1841), 2 tomes, tome 2, pp. 157-159 ; L'Atelier, n 4, janvier1844, p. 62 ; Jeffry

KAPLOW, art. cité, p. 117.

127.. Émile LE ROY, La Saint-Lundi. Pièce réalisée en un acte, Châlons-sur-Marne, Le Roy, 1885.

128.. Cité par Daniel RONDEAU, Chagrin lorrain : la vie ouvrière en Lorraine, 1870-1914, Paris, Éditions du Seuil, 1979, p. 141. 
129.. Même si le repos dominical est observé dans la plus grande partie de l'industrie française depuis les années 1880 , suite à la crise économique, mais aussi suite à de nouvelles conceptions hygiéniques et morales (Robert BECK, ouv. cité, pp. 269-276). Mais il s'agit de l'obtenir pour les délaissés du repos dominical, les employés de commerce surtout. À ce sujet, voir notre article, " "C'est dimanche qu'il nous faut". Les mouvements sociaux en faveur du repos dominical et hebdomadaire en France avant 1906 ", dans Le Mouvement social, $\mathrm{n}^{\circ}$ 184, juillet-septembre 1998, pp. 23-51. Suite à ce mouvement social, le législateur accorde finalement le repos hebdomadaire à l'ensemble de monde salarial dans la loi du 13 juillet 1906. Cette loi, dont l'application s'avérera assez compliquée, est liée à de nombreuses dérogations, dont celle de transférer le repos hebdomadaire des employés au lundi, dont le commerce fait surtout usage. Or ce repos du lundi ne possède aucune ressemblance avec la défunte Saint Lundi des artisans et ouvriers.

\section{RÉSUMÉS}

Durant une grande moitié du XIX ${ }^{\mathrm{e}}$ siècle, la coutume de chômer le lundi, appelée la Saint Lundi, prend une nouvelle extension en France dans le cadre de la première industrialisation. Cette coutume qui existe surtout dans l'industrie à caractère artisanal, change alors de caractère : suite au développement du travail du dimanche, le lundi chômé devient un temps autonome qui sert aussi progressivement aux activités politiques et syndicales. C'est cette dernière circonstance qui vaut à cette coutume, désapprouvée depuis des siècles, les foudres des élites religieuses, économiques, moralistes et philanthropes. En lui opposant les vertus du travail, de la famille, de la sobriété et de l'épargne, celles-ci vont la combattre énergiquement, surtout après la Commune. Mais la quasi-disparition de la Saint Lundi vers la fin du XIX ${ }^{\mathrm{e}}$ siècle s'explique aussi par la désaffection d'une grande partie de la classe ouvrière pour cette coutume dorénavant mal famée. Elle lui préfère le repos du dimanche, jour de famille, de loisirs et, désormais, d'activités politiques et syndicales.

The Golden Age and the decline of Saint Monday in $19^{\text {th }}$ century France. During more than half of $19^{\text {th }}$ century, the habit of not working on mondays, called Saint Monday, could be found throughout France with the advent of the industrialized society, above all among craftsmen. It changed with the habit of working on Sundays, which gave to the time of Monday an independent character, a time not only for drinking but also for political and trade-union activities. This is the reason why this old habit, having been disapproved of for centuries, is now sharply criticized by religious, economic, moralistic and philanthropist elite. This elite used a discourse about the virtues of labour, family, temperance and savings against Saint Monday and fought against it, particularly after the Paris Commune. But the near extinction of Saint Monday at the end of the $19^{\text {th }}$ century can also be explained by increasing disaffection of many in the working class who preferred the Sunday rest - a family day for leisure, cultural, and now also for political and trade-union activities. 


\section{AUTEUR}

ROBERT BECK

maître de conférences à l'Université François Rabelais de Tours 